\title{
Building Public Health System Capacity for Diabetes and Hypertension Care in Western Kenya
}

\author{
Michael Harding ${ }^{1}$, Matthew Turissini ${ }^{1234}$ \\ ${ }^{1}$ Indiana University School of Medicine, Indianapolis, IN, USA; ${ }^{2}$ Academic Model Providing Access to Healthcare, \\ Eldoret, Kenya; ${ }^{3}$ Department of Medicine, Moi University School of Medicine, Eldoret, Kenya; ${ }^{4}$ Moi Teaching and \\ Referral Hospital, Eldoret, Kenya
}

\section{Background and Hypothesis:}

In western Kenya, estimates for prevalence of hypertension are 6-24\% and diabetes are 3-5\%. Complications of hypertension and diabetes are some of the fastest growing causes of morbidity and mortality in Kenya. There is limited knowledge and poor training pertaining to noncommunicable diseases (NCDs) making treatment difficult. With shortages of physicians and mid-level providers, protocol driven nursing care of hypertension and diabetes has shown to be effective. Through partnerships with counties, the NCD care program has screened 134,923 and treated 12,566 individuals for diabetes and screened 238,078 and treated 29,377 individuals for hypertension.

My summer project was to utilize Academic Model Providing Access to Healthcare's (AMPATH) partnership with the Kenyan Ministry of Health $(\mathrm{MOH})$ to improve access and quality of hypertension and diabetes care delivery at county $\mathrm{MOH}$ facilities. These improvements can be achieved through increasing our joint mentorship program led by AMPATH and county MOH staff; introducing the AMPATH Medical Record System (AMRS) into county clinics to improve care; and quality improvement with the Home Glucose Monitoring team.

\section{Personal Role:}

I created Standard Operating Procedures (SOPs) for Integrative Screening, Clinical Mentorship, and Home Glucose Monitoring (HGM); designed a user manual for AMRS; helped develop a M\&E plan for the HGM program; and assisted in the authorship of a grant to scale mentorship for diabetes and hypertension to 60 more clinical sites.

\section{Conclusion and Potential Impact:}

This project is a product of the partnership of AMPATH and the MOH to make accessible, highquality care for hypertension and diabetes available across levels of care. By partnering with the Kenyan government and implementing care in the public sector, improvements in care are sustainable. My involvement will facilitate scaling the project to treat more patients through improving organizational capacity and helping apply for funds to implement in new areas. 